\title{
Solvation in Ionic Liquids with Polymer-Grafted Nanoparticles
}

\author{
Siqi Liu ${ }^{\dagger}$, Mia Walton ${ }^{\dagger}$, Nadezda V. Tarakina ${ }^{\S}$ and Pinar Akcora* ${ }^{\dagger}$ \\ ${ }^{\dagger}$ Department of Chemical Engineering \& Materials Science, Stevens Institute of Technology \\ Hoboken, NJ 07030, USA \\ ${ }^{\S}$ Department of Colloid Chemistry, Max Planck Institute of Colloids and Interfaces \\ Research Campus Golm, Potsdam 14476, Germany
}

*Corresponding author: pakcora@stevens.edu, Tel: (201) 216-5060

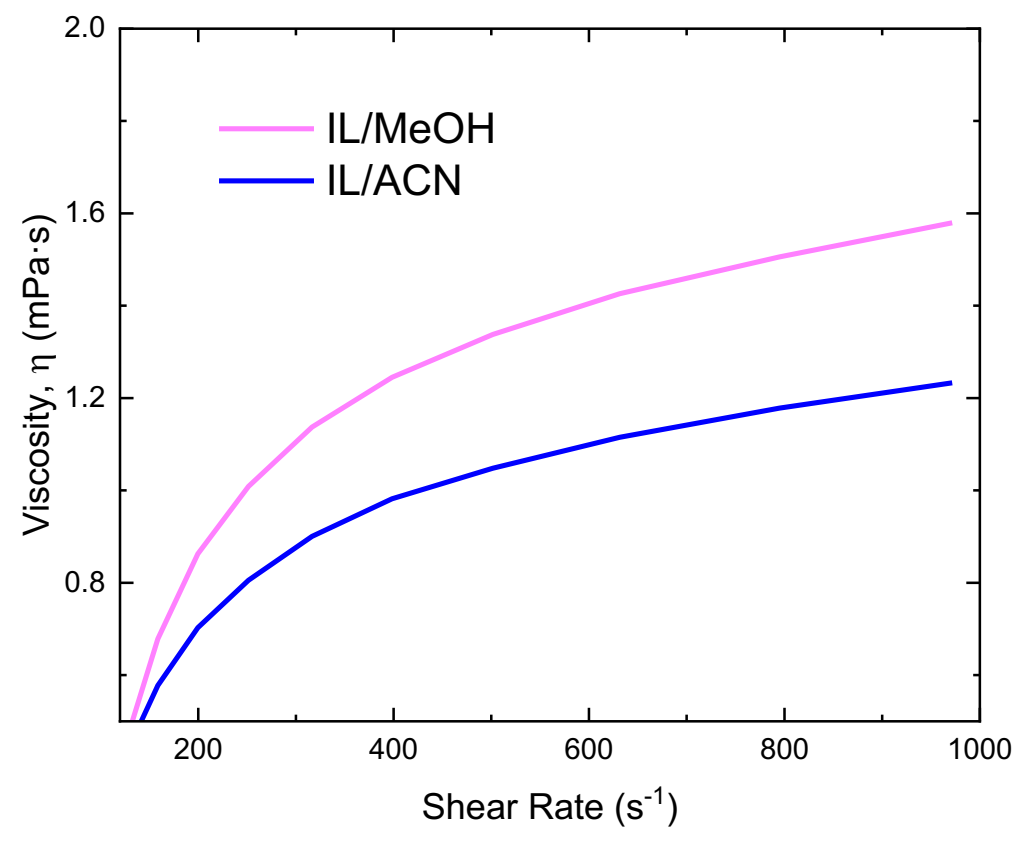

Figure S1. Viscosity measured at varying shear rate for IL/methanol (MeOH) and IL/acetonitrile (ACN) mixtures. IL: HMIM-TFSI in all labels. 


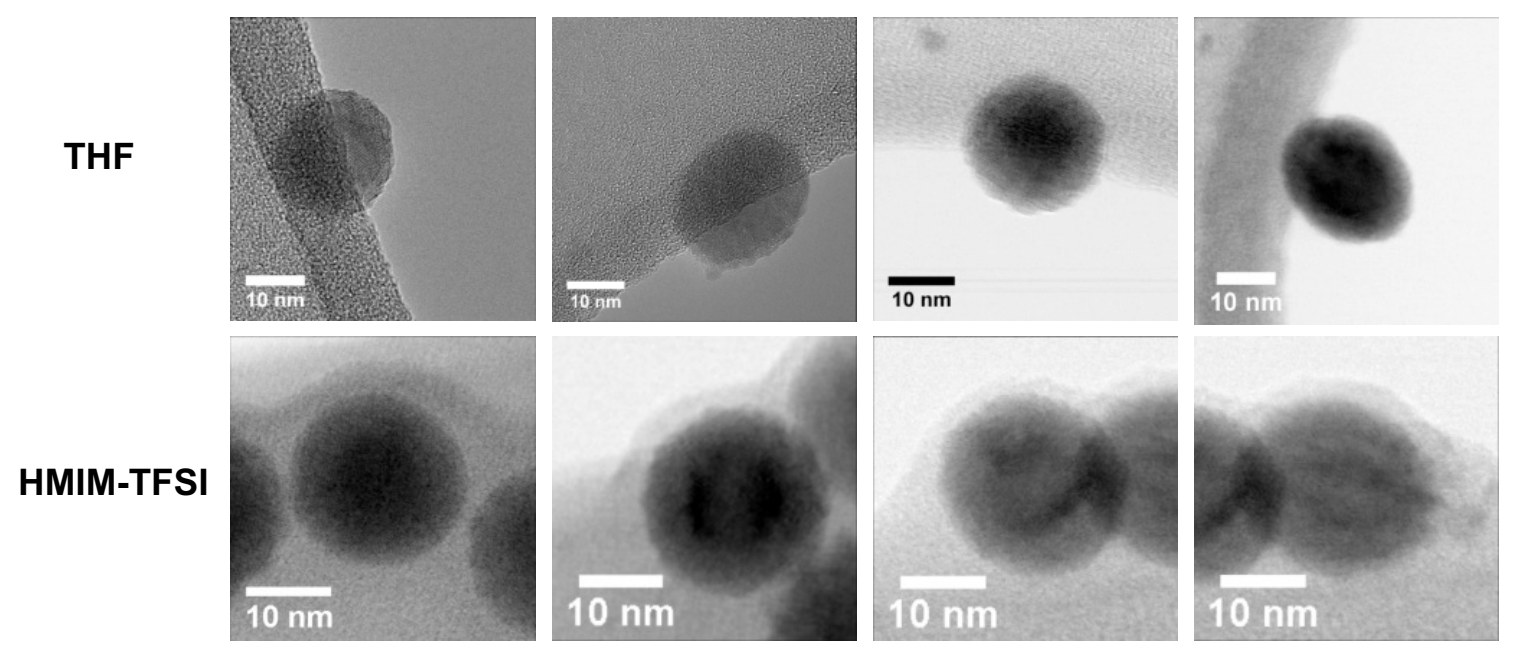

Figure S2. TEM images of bare particles solution cast from either THF or HMIM-TFSI (as labeled for each row) used in corona thickness analysis.

Roughness Analysis. Particle surface roughness was obtained following the reported procedure. Surface roughness of PMMA-grafted nanoparticles was analyzed by processing the TEM images in MATLAB. In the Image Segmenter App, a mask was applied through the "flood fill" tool to a raw TEM image and the corona boundary was accurately isolated from any background noise through the "Morphology" operation. The corresponding binary image was generated simultaneously and then imported into MATLAB. Through a self-developed script, more than 1000 points in the X-Y coordinates (Figure S3d) along the corona boundary (highlighted in red in Figure S3c) for every single particle were obtained. Distances from the particle center to the corona boundary were calculated after entering the particle core coordinates as inputs, which were then plotted against interior angle (Figure S3e) to get the boundary profile for each sample. An angle of $0^{\circ}$ was chosen as the starting position of the corona boundary. The roughness of a particle was calculated from the averaged difference of maximum and minimum distance for each $30^{\circ}$ interval $^{1}$. 


\section{TEM image}
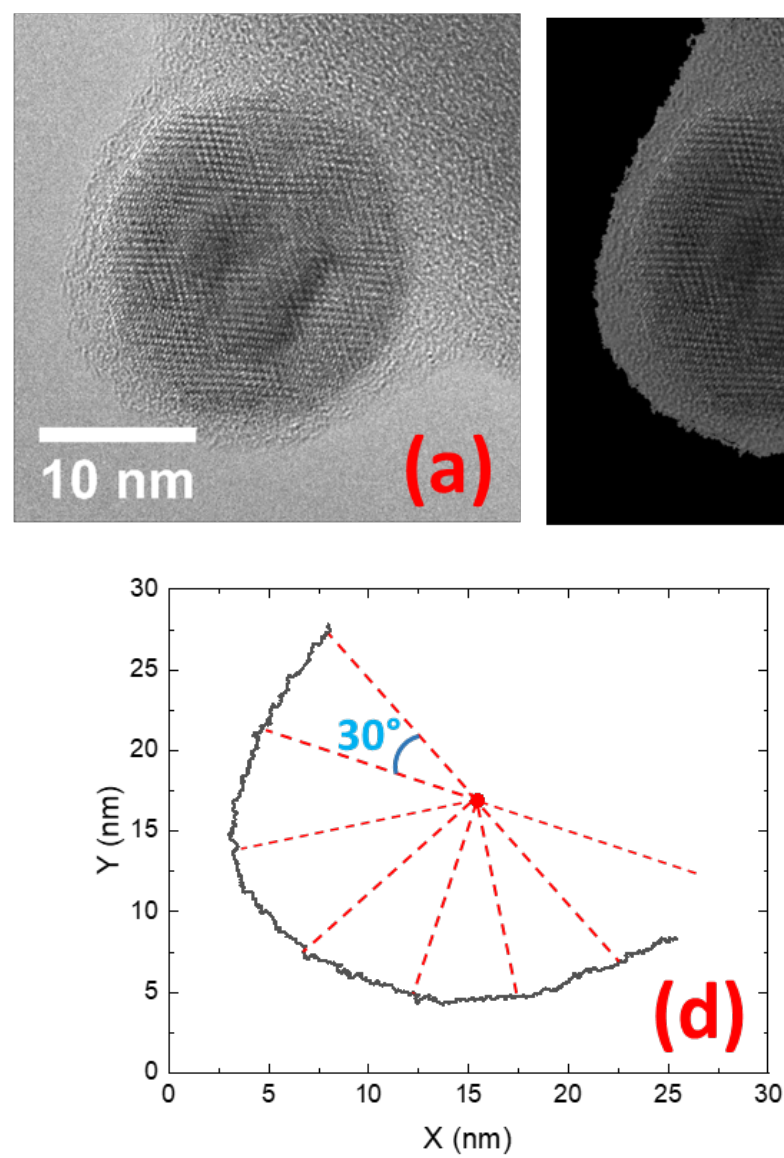

Mask applied
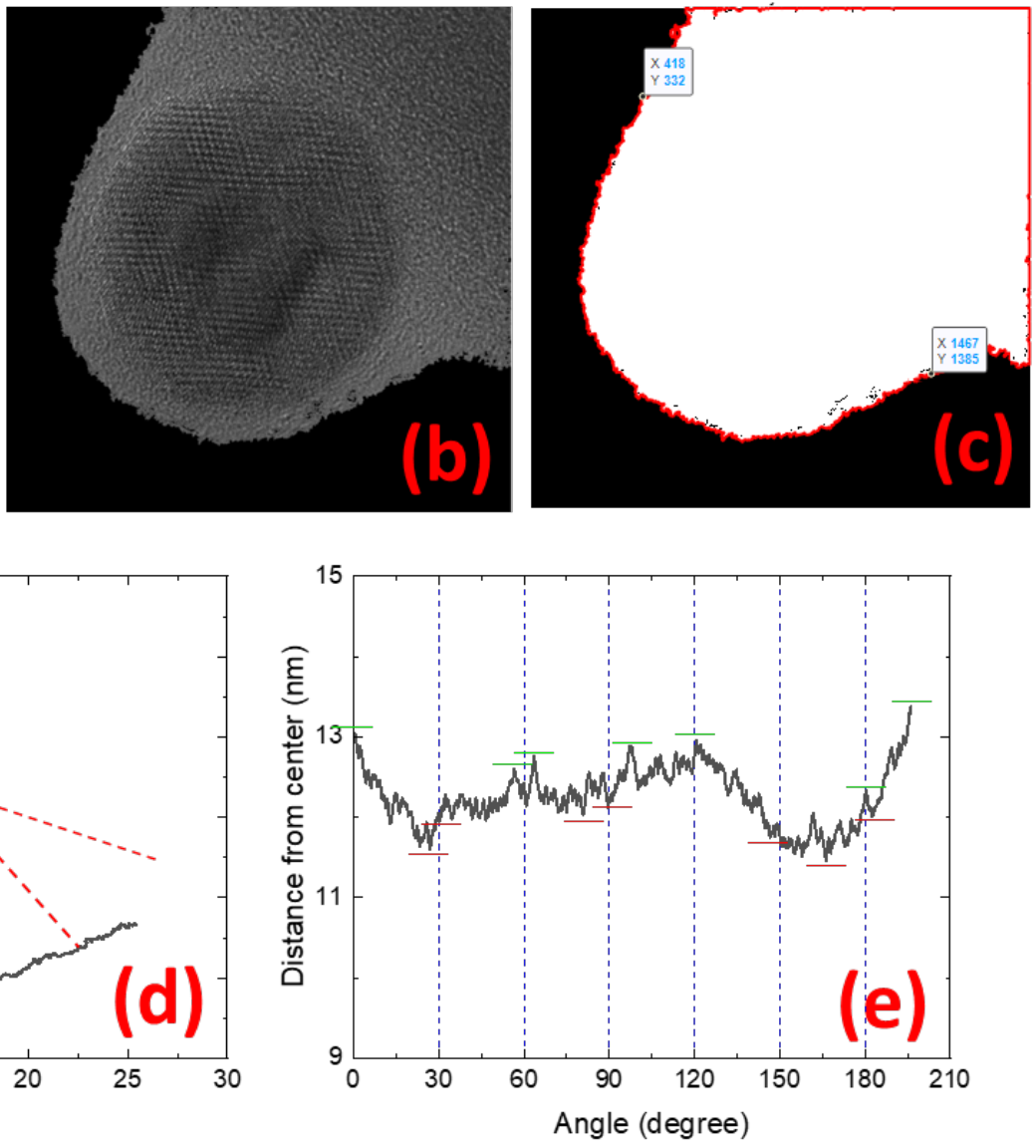

Figure S3. a) TEM image of a PMMA-grafted $\mathrm{Fe}_{3} \mathrm{O}_{4}$ particle (122 $\mathrm{kDa}-0.7$ chains $/ \mathrm{nm}^{2}$ in HMIMTFSI/methanol); b) mask application after "flood fill" and "morphology" operation in Image Segmenter of Matlab; c) X-Y coordinates along the corona boundary; d) X-Y plot of the selected boundary range divided into $30^{\circ}$ interval; e) boundary profile calculated from (d). At each $30^{\circ}$ interval, the maximum and minimum distance from boundary to center was labeled with green and red colors, respectively. 


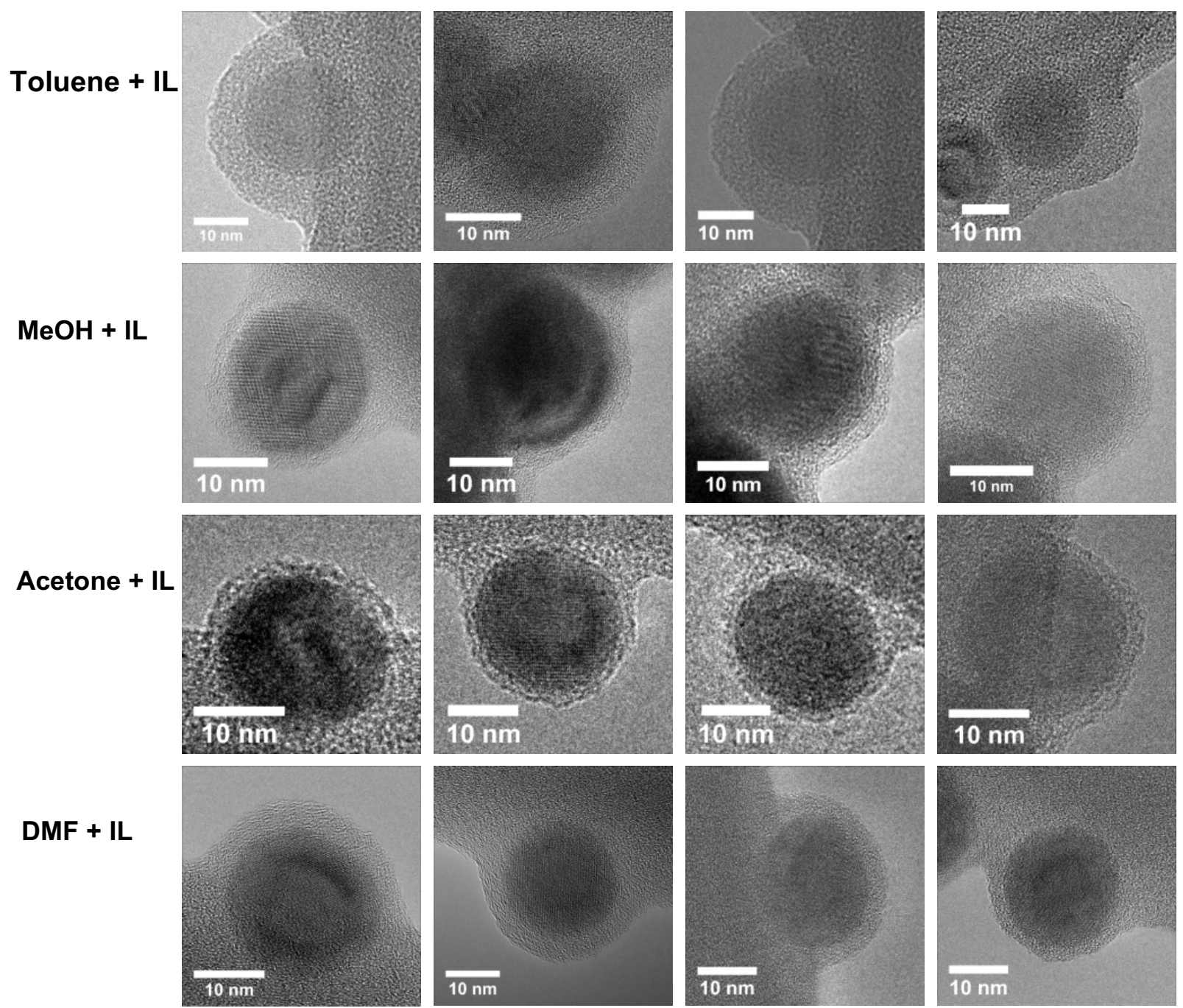

Figure S4. TEM images of $122 \mathrm{kDa}-0.7$ chains $/ \mathrm{nm}^{2}$ in HMIM-TFSI/solvent mixtures (labeled for each row) used for surface roughness analysis. IL: HMIM-TFSI in all labels. 


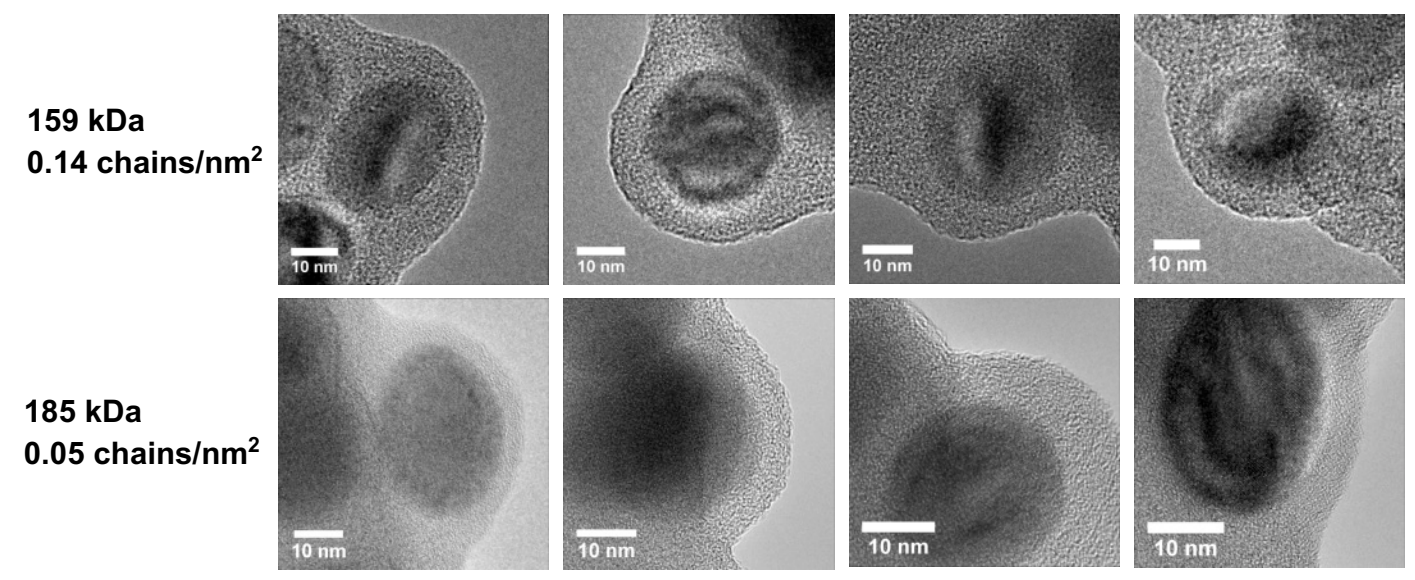

Figure S5. TEM images of $159 \mathrm{kDa}-0.14$ chains $/ \mathrm{nm}^{2}$ and $185 \mathrm{kDa}-0.05$ chains $/ \mathrm{nm}^{2}$ in HMIMTFSI/acetone mixtures used for surface roughness analysis in Figure 3.

(a)

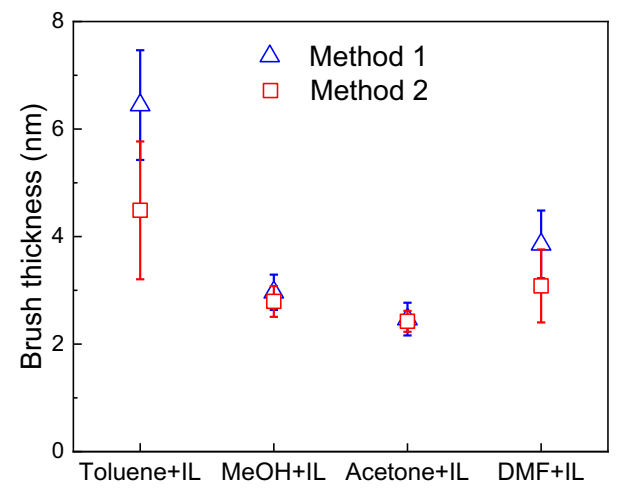

(b)

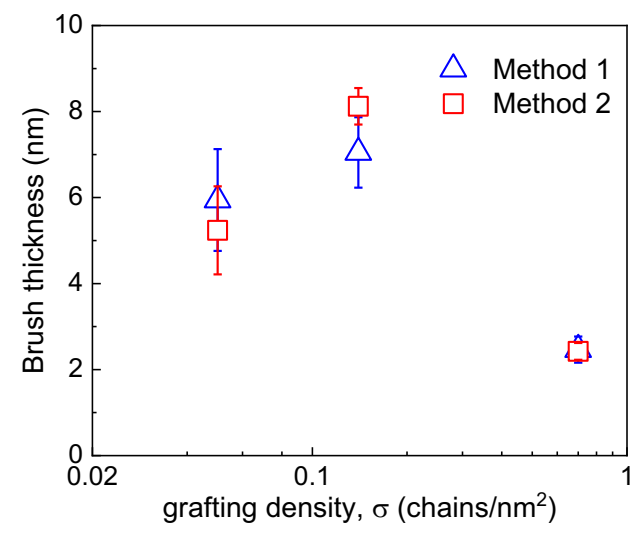

Figure S6. Comparison of brush thickness between two methods calculated from roughness analysis (Method 1) and averaging over several particles (Method 2) a) PMMA-grafted particles (122 kDa, 0.7 chains $/ \mathrm{nm}^{2}$ ) cast from IL-solvent mixtures, b) PMMA-grafted nanoparticles (185 kDa with 0.05 chains $/ \mathrm{nm}^{2}, 159 \mathrm{kDa}$ with 0.14 chains $/ \mathrm{nm}^{2}$ and $122 \mathrm{kDa}$ with 0.7 chains $/ \mathrm{nm}^{2}$ ) cast from IL/acetonitrile (ACN) mixtures. IL: HMIM-TFSI in all labels. 


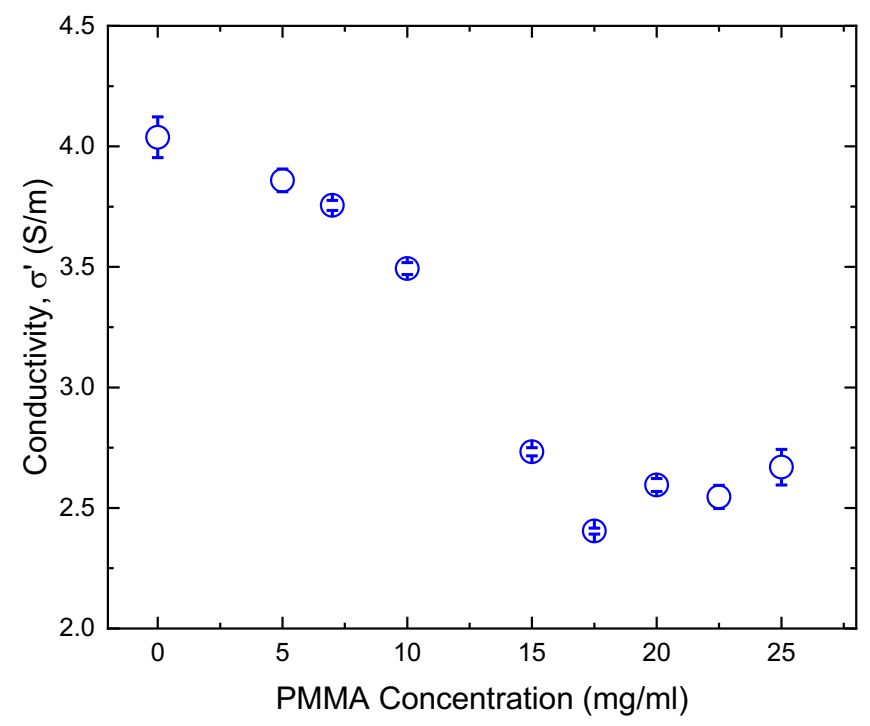

Figure S7. Ionic conductivity of HMIM-TFSI/acetonitrile mixture with various PMMA (118 kDa) homopolymer concentrations.

(a)

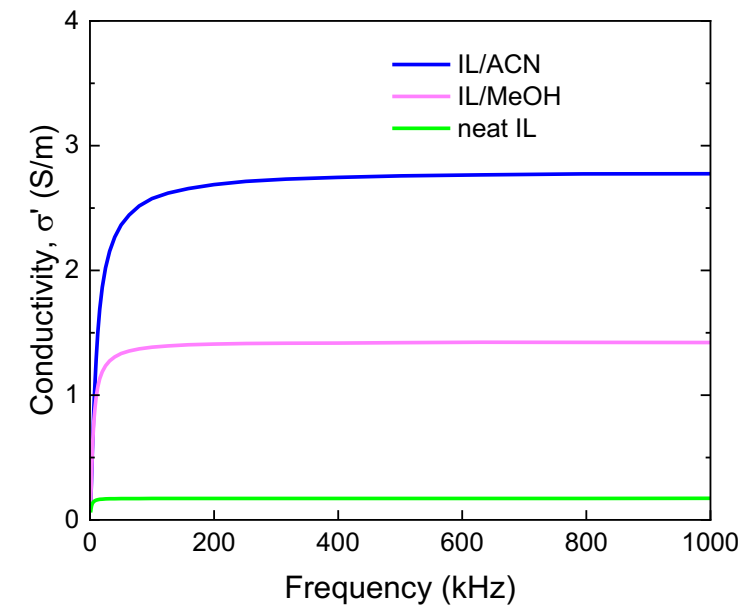

(b)

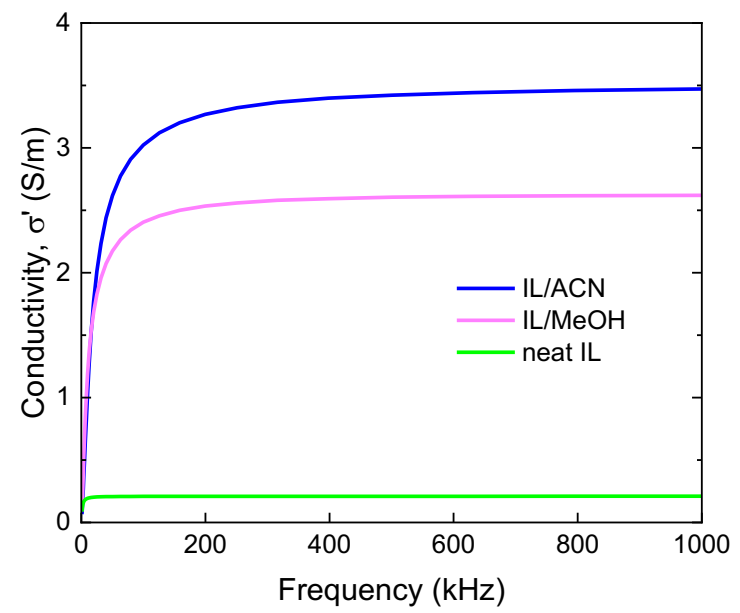

Figure S8. Real part of complex conductivity of grafted nanoparticles with a) $42.2 \mathrm{kDa}$ PMMA and 0.14 chains $/ \mathrm{nm}^{2}$ and b) $31.2 \mathrm{kDa}$ PMMA and 0.88 chains $/ \mathrm{nm}^{2}$ dissolved in pure IL, IL/acetonitrile (ACN) and IL/methanol (MeOH) mixtures. IL: HMIM-TFSI in all labels. 

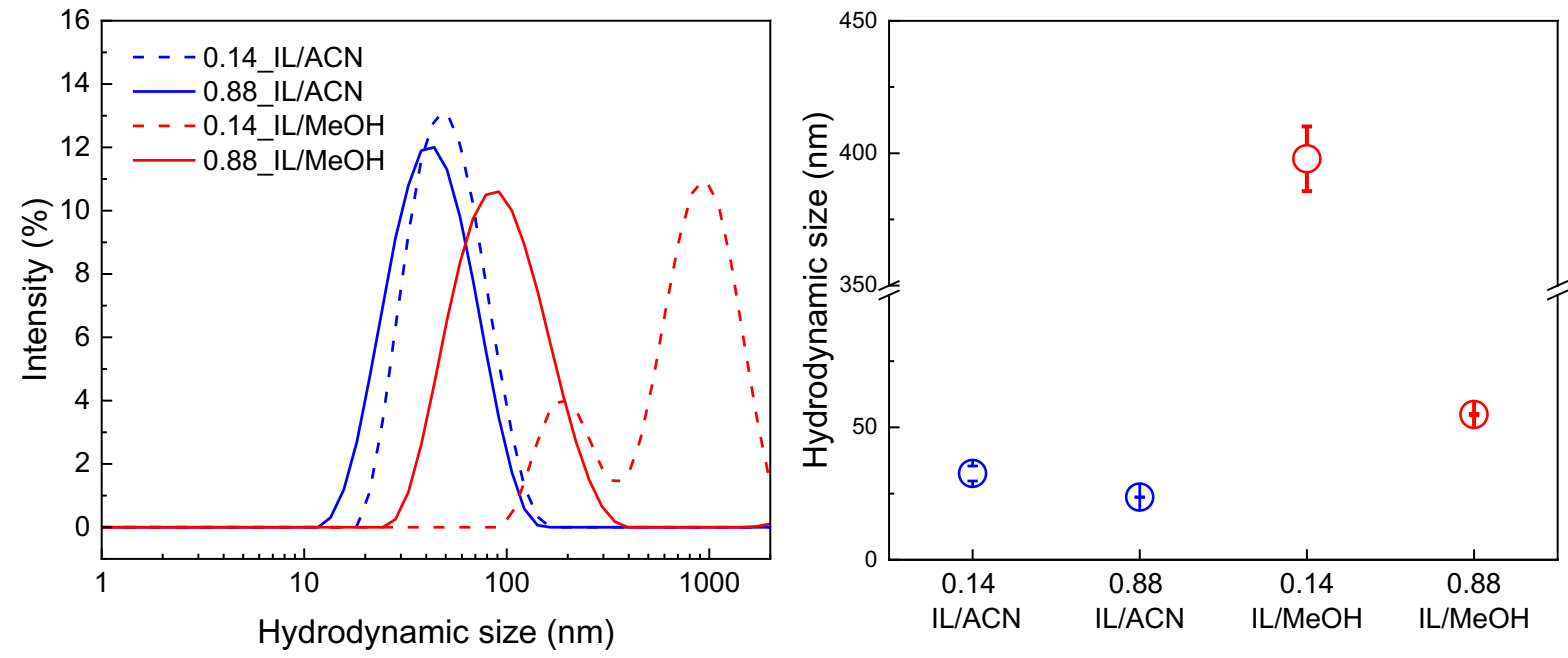

Figure S9. Hydrodynamic size distributions and averaged hydrodynamic sizes of grafted nanoparticles for $42.2 \mathrm{kDa}$ PMMA with 0.14 chains $/ \mathrm{nm}^{2}$ and $31.2 \mathrm{kDa}$ PMMA with 0.88 chains $/ \mathrm{nm}^{2}$ in IL/acetonitrile $(\mathrm{ACN})$ and IL/methanol (MeOH) mixtures. IL: HMIM-TFSI in all labels.

\section{References}

1. Wang, Y. C.; Slater, T. J. A.; Rodrigues, T. S.; Camargo, P. H. C.; Haigh, S. J., Automated quantification of morphology and chemistry from STEM data of individual nanoparticles. Journal of Physics: Conference Series 2017, 902, 012018. 